\title{
PART 2: CAN IMPROVEMENT IN OUTCOME MEASUREMENT IN PLASTIC SURGERY BE ACHIEVED IN TODAY'S HEALTH SYSTEMS?
}

\author{
Luke Geoghegan $^{1}$, Thomas Dobbs ${ }^{2}$, Jeremy N Rodrigues ${ }^{1}$ \\ ${ }^{1}$ Nuffield Department of Orthopaedics, Rheumatology and Musculoskeletal Sciences, University \\ of Oxford, Botnar Research Centre, Nuffield Orthopaedic Centre, Windmill Road, Oxford, UK \\ ${ }^{2}$ Reconstructive Surgery \& Regenerative Medicine Research Group, Institute of Life Sciences, \\ Swansea University Medical School, Swansea, UK.
}

Being able to accurately measure relevant constructs is particularly valuable in plastic surgery, where the objective of treatment may range across improving hand function, identifying satisfaction with breast reconstruction, or establishing quality of life changes from surgery. This matters in research applications such as clinical trials, and for quality assurance of surgery through routine data collection in clinical practice. The technical aspects of refining measurement to ensure that it is accurate and the process for selecting outcome measures have both been described in recent articles in this journal., ${ }^{1,2}$ Furthermore, the potential breadth of the practical applications of outcome measurement has been discussed in the accompanying editorial ("Part 1"). If such improved measurement is expected to benefit patients and clinicians, we must establish how optimised measurement can gain traction in health systems.

There are some mechanisms involving outcome measurement that are mandatory. While mandatory data collection might be construed as undesirable by some clinicians, it also has potential advantages. These advantages may apply at the microsystem level of the individual surgeon or department, or across the health service in general. In contrast, when there is no mandate to drive routine outcome measurement, generating buy-in to data collection is key. 
There are examples in the United Kingdom National Health Service (NHS) where outcome measurement has been mandated. With the aim of determining the effectiveness of patient care and service provision, NHS England has established the collection of patient-reported outcome measures (PROMs) following hip and knee replacement, varicose vein surgery and groin hernia repair through the National PROMs Programme. ${ }^{3}$ PROM data for arthroplasty also contributes to The National Joint Registry (NJR). The NJR collects surgical, functional and patient-reported outcomes to determine the effectiveness of interventions and improve national clinical standards for hip and knee replacement. ${ }^{4}$ At surgeon or individual centre level, the NJR permits reflective practice and enables the impact of changes to care pathways to be studied. Data can also be used to support revalidation and other quality assurance processes with minimal additional burden on the surgeon at the time of accessing data. The fact that this data collection is mandatory probably explains much of the disparity in completion rate between hip and knee PROMs (mandatory) and shoulder PROMs (not mandatory) in the NJR. ${ }^{5}$ As participation is compulsory, resources are allocated to data collection and processing; in the absence of this, individuals might have to collect data without financial or administrative support. Mandatory outcome measurement may expand in plastic surgery too. The Royal College of Surgeons of England has proposed compulsory collection of PROM data for cosmetic surgery. ${ }^{6}$ It is also conceivable that the use of existing registries in plastic surgery may also become compulsory. In the UK, our specialty already has a UK National Hand Registry and a UK National Flap Registry provided by the British Society for Surgery of the Hand and the British Association of Plastic, Reconstructive and Aesthetic Surgeons respectively. ${ }^{7,8}$ If such systems adopt the quality outcome measures and capture a large proportion of the treatment delivered in a system, then formal recognition of their role may follow.

There are other aspects of health service policy that could also act as levers to support effective measurement in clinical practice. These include the NHS Outcomes Framework (NOF), the Commissioning for Quality and Innovation (CQUIN) national goals, and the 
National Institute for Health and Care Excellence (NICE) Quality Standards. NOF comprises domains spanning clinical effectiveness, patient experience and safety through which local commissioners should commission on the basis of improved outcomes. It is one process for holding NHS Commissioning Board accountable for the health of the nation. ${ }^{9}$ CQUIN mandates quality improvement through incentivising demonstrated improvements in quality and innovation in specified areas of patient care. ${ }^{10}$ Quality Standards represent evidence based markers of cost-effective care for defined conditions. However, currently there is a limited number of outcome measures that can be used as measures of clinical quality. ${ }^{11}$ While such processes may incentivise or drive data collection, they have to cover all areas of health care, and plastic surgery may not be prioritised in them.

Where there is no formal mandate to measure outcomes routinely, generating buy-in by stakeholders (including clinicians, patients, and others) is essential if participation and completion is to be high. Some steps towards achieving buy-in include demonstrating the importance and value of data, standardising the use of the best-available outcome measures, ensuring that high quality data are translated into practice through clinical guidelines, and reducing the burden of data collection.

Priority setting partnerships (PSPs) bring together surgeons and other clinicians, patients and healthcare providers to identify and prioritise uncertainties. They may be run by organisations like The James Lind Alliance. ${ }^{12}$ Research priorities that arise from PSPs are validated as being of importance to all represented stakeholders. As well as drawing the attention of major research funders like the National Institute for Health Research (NIHR), they should reiterate the importance of collecting outcome data in these areas in research and potentially in routine practice too. PSPs have been developed for some plastic surgery subspecialties like common hand conditions, cleft lip and palate, and hidradenitis suppurativa. ${ }^{13}$ Other areas of plastic surgery may also benefit if further PSPs are conducted. 
If the importance of an area of uncertainty has been highlighted by a PSP, then research into the question should employ the best outcome measurement tools available. Assessing the quality of outcome measures like PROMs can be supported by using existing criteria, such as those provided by the Consensus-based Standards for the selection of health status Measurement Instruments (COSMIN) initiative. ${ }^{14}$ Such methods of outcome measurement validation have been discussed and summarised recently. ${ }^{1}$

Even if guidance is followed, there is still likely to be variation in measurement, and this is often undesirable. ${ }^{15}$ Standardisation of outcome measurement could reduce variation, particularly when routine data collection across one or more countries is to be implemented. Core outcome sets (COS) provide a recommended minimum dataset that should be measured and reported when studying a specific condition. ${ }^{16}$ There can be variation in the methods used to generate COS. The International Consortium for Health Outcomes Measurement (ICHOM) is one organisation working with stakeholders to develop and implement globally-relevant standard sets of outcome measurements, each specific to a medical condition. ${ }^{17}$ Recognition of the need for COS has stimulated the development of COMET (Core Outcome Measures in Effectiveness Trials) Initiative, ${ }^{18}$ which facilitates the development and dissemination of COS. Outcome measurement is an evolving field, and COS may need to be revised as PROMs with better methodological quality are developed.

Once outcome measurement has been refined and standardised, the burden of data collection can be reduced and waste can be avoided to make routine outcome measurement more achievable. Simplifying data collection and then routinely collecting data in our registries may help. The burden of data collection can also be reduced by techniques such as computerised adaptive testing (CAT). A CAT involves using a computer to administer an appropriate PROM, one question at a time. Using an algorithm, the CAT chooses the subsequent question based on the previous answer given. By selecting only the questions that will provide more information, the person's score can be determined without having to 
ask all of the questions. CATs and standard electronic PROMs could be administered by virtual assistants (such as Siri or Alexa, or similar) to further reduce the manpower required for data collection. If the burden of data collection is reduced through steps like these, then routinely collecting clinical practice data in registries is likely to be more achievable, even when it is not mandatory.

Sharing the load of designing and conducting research through multi-centre collaboration is also beneficial. This may be through project-specific arrangements, or through established collaboratives like The Reconstructive Surgery Trials Network (RSTN).

If efforts are made to collect high quality routine data, in either research studies or in routine clinical practice, then incorporating this evidence into guidelines may create greater impact from these endeavours. Guidelines that meet the standards set in tools like Appraisal of Guidelines for Research and Evaluation (AGREE) II, ${ }^{19}$ may align with health service policy levers, leading to better implementation of their recommendations. For example, in the UK, AGREE II forms the basis for Accreditation of guideline developers by the National Institute for Health and Care Excellence (NICE), with Accredited guidelines able to inform NICE's Quality Standards, which have been discussed above.

These are some of the potential pathways through which outcome measurement could be improved, facilitated and translated into service improvement. Some of these options may not be able to be harnessed. Furthermore, the exact processes and policies concerned will vary between countries and between health services, and may change over time. However, fostering a culture of high-quality measurement in plastic surgery, encouraging enthusiasm for data collection in everyday clinical practice as well as in research, and being prepared to embrace innovations in measurement science will all contribute to better understanding of the reality of the conditions we treat, and the true impacts of the interventions that we offer. 
Funding statement: JR is funded by the National Institute for Health Research (NIHR).

This article presents independent research funded by the NIHR. TD is funded by the Welsh

Clinical Academic Training (WCAT) Fellowship. The views expressed are those of the authors and not necessarily those of the NHS, the NIHR or the Department of Health.

\section{REFERENCES}

1. Wormald JCR, Rodrigues JN. Outcome Measurement in Plastic Surgery. J Plast Reconstr Aesthet Surg 2018;71;283-89.

2. Dobbs T, Hughes $\mathrm{S}$, Mowbray $\mathrm{N}$, Hutchins $\mathrm{H}$, Whitaker I. (in press) How to decide which patientreported outcome measures to use? A practical guide for plastic surgeons. J Plast Reconstr Aesthet Surg 2018; doi: 10.1016/j.bjps.2018.03.007

Hutchings HA, Whitaker IS. UK-based Prospective Cohort Study to Anglicise and Validate the FACE-Q Skin Cancer Module in Patients with Facial Skin Cancer Undergoing Surgical Reconstruction: the PROMISCR (Patient-Reported Outcome Measure in Skin Cancer Reconstruction) Study. BMJ Open 2017: 7: e016182.

3. NHS England. National Patient Reported Outcome Measures (PROMs) Programme Consultation. https://www.engage.england.nhs.uk/consultation/proms-programme/ [Accessibility verified March $27,2018]$

4. National Joint Registry for England, Wales and Northern Ireland. About the NJR. http://www.njrcentre.org.uk/njrcentre/AbouttheNJR/tabid/73/Default.aspx [Accessibility verified March 27, 2018]

5. National Joint Registry for England, Wales and Northern Ireland. $14^{\text {th }}$ Annual Report. http://www.njrcentre.org.uk/njrcentre/Reports,PublicationsandMinutes/Annualreports/tabid/86/D efault.aspx. [Accessibility verified March 13, 2018]

6. The Royal College of Surgeons of England. Patient Reported Outcome Measures. https://www.rcseng.ac.uk/standards-and-research/standards-and-guidance/servicestandards/cosmetic-surgery/clinical-quality-and-outcomes/patient-reported-outcome-measures/ [Accessibility verified March 27, 2018]

7. The British Society for Surgery of the Hand. The UK Hand Registry. https://www.ukhr.net/ [Accessibility verified March 27, 2018]

8. The British Association of Plastic Reconstructive and Aesthetic Surgeons. UK National Flap Registry. https://hscn.e-dendrite.com/csp/bapras/frontpages/index.html [Accessibility verified March 27, 2018]

9. Clinical Indicators Team, NHS Digital. NHS Outcomes Framework, England, August 2017 Quarterly Publication. http://digital.nhs.uk/catalogue/PUB30062 [Accessibility verified March 13, 2018] 
10. NHS England. Commissioning for quality and innovation (CQUIN) guidance for $2017 / 19$. Leeds: Department of Health. 2015.

11. National Institute of Clinical Excellence. Standards and Indicators.

https://www.nice.org.uk/standards-and-indicators [Accessibility verified March 13, 2018]

12. Cowan K, Oliver S. The James Lind Alliance Guidebook. Southampton, UK: National Institute for Health Research Evaluation, Trials and Studies Coordinating Centre. 2013.

13. The James Lind Alliance. The PSPs. http://www.jla.nihr.ac.uk/priority-setting-partnerships/ [Accessibility verified April 2, 2018]

14. Mokkink LB, Terwee CB, Patrick DL, et al. The COSMIN study reached international consensus on taxonomy, terminology, and definitions of measurement properties for health-related patientreported outcomes. J Clin Epidemiol 2010: 63: 737-45.

15. Ball C, Pratt AL, Nanchahal J. Optimal functional outcome measures for assessing treatment for Dupuytren's disease: a systematic review and recommendations for future practice. BMC Musc Disord 2013; 14(1);131

16. Core Outcome Measures in Effectiveness Trials. COMET Initiative. http://www.cometinitiative.org/ [Accessibility verified March 27, 2018]

17. Kelley TA. International Consortium for Health Outcomes Measurement (ICHOM). Trials. 2015;16(3);04.

18. Prinsen CA, Vohra S, Rose MR, et al. Core Outcome Measures in Effectiveness Trials (COMET) initiative: protocol for an international Delphi study to achieve consensus on how to select outcome measurement instruments for outcomes included in a 'core outcome set'. Trials 2014;15; 247.

19. AGREE Next Steps Consortium. Appraisal of guidelines for research and evaluation II: AGREE II instrument. http://www.agreetrust.org/wp-content/uploads/2013/10/AGREE-II-Users-Manual-and23-item-Instrument 2009 UPDATE 2013.pdf. [Accessibility verified March 11, 2018] 